\title{
Thymosin $\beta 4$ promotes angiogenesis and increases muscle progenitor cell density in ischemic skeletal muscle in a mouse model of hind limb ischemia
}

\author{
Yue Zhou ${ }^{1}$, Eliana C. Martinez ${ }^{2}$, Li-Ping Su ${ }^{3}$, Kok-Onn Lee ${ }^{1}$, Lei Ye ${ }^{1,4}$ \\ ${ }^{1}$ Department of Medicine, National University of Singapore, Singapore City, Singapore \\ ${ }^{2}$ Department of Surgery, National University of Singapore, Singapore City, Singapore \\ ${ }^{3}$ Division of Bioengineering, National University of Singapore, Singapore City, Singapore \\ ${ }^{4}$ Department of Medicine, University of Minnesota, Minneapolis, USA; yeleislp@yahoo.com
}

Received 8 July 2012; revised 17 August 2012; accepted 13 September 2012

\section{ABSTRACT}

Aim: To determine the therapeutic effect of thymosin $\beta 4$ (T/34) for treatment of ischemic limb disease in a mouse model. Methods: A mouse model of hindlimb ischemia was created by permanent ligation of femoral arteries and internal iliac artery. T $\beta 4$ was dissolved in sterile saline and intramuscularly injected into the centre and periphery of ligation area in the treatment group $(n=10)$ starting from the surgery day until 4 weeks after surgery, while control animals received saline injection only $(n=9)$. All animals were sacrificed at 6 weeks after surgery and used for immunohistochemistry studies. Results: T $\beta 4$ stimulated angiogenesis was evidenced by increased vascular density based on CD31 immunostaining, which was signifycantly increased in T $\beta 4$ group $(562.5 \pm$ $78.4 / \mathrm{mm}^{2}$ ) as compared with control group $\left(371.1 \pm 125.7 / \mathrm{mm}^{2} ; p<0.05\right)$. The arteriole density based on CD31 and SMA dual immunostaining was similar between the T $\beta 4$ (27.2 \pm $\left.16.9 / \mathrm{mm}^{2}\right)$ and control $\left(35.3 \pm 6 / \mathrm{mm}^{2} ; p>0.05\right)$ groups. T $\beta 4$ increased $\mathrm{Pax} 3 / 7^{+}$skeletal muscle progenitor cell density. Pax $3 / 7^{+}$cell density of $\mathrm{T} \beta 4$ group $(13.7 \% \pm 2 \%)$ was significantly higher than that of the control group $(4.3 \% \pm 1.6 \%, p<$ $0.05)$. However, the numbers of central nuclei fiber and central nuclei per fiber were insignificantly increased in T $\beta 4$ group as compared to control group. The numbers of central nuclei fiber were $8.9 \pm 2.1$ and $9.5 \pm 1.6$, and the central nuclei per fiber were $0.25 \pm 0.07$ and $0.48 \pm 0.09$ for control and T $\beta 4$ groups, respectively. Conclusions: This preliminary study suggests that localized delivery of T $\beta 4$ increased angiogenesis and skeletal muscle progenitor cell density in ischemic skeletal muscle, but failed to promote skeletal muscle regeneration.

Keywords: Thymosin $\beta 4$; Neovascularization; Progenitor Cell; Muscle Regeneration

\section{INTRODUCTION}

Thymosin $\beta 4$ (T $\beta 4$ ), a ubiquitous 43 amino acid, is a 5 $\mathrm{kDa}$ polypeptide that plays an important role in mediating cell survival, migration, and proliferation [1]. It is the most abundant member of the $\beta$-thymosin family in mammalian tissue and is regarded as the main G-actin sequestering peptide [1]. The role of $\mathrm{T} \beta 4$ in promoting cell survival, migration and proliferation has been extensively studied [2-4]. For instance, T $\beta 4$ promotes cardiac cell survival, migration and proliferation after an episode of myocardial infarction $\mathrm{T} \beta 4$ induces hair follicle stem cell migration that contributes to new hair growth [2-4]. It promotes neuron survival and neurite outgrowth of cultured spinal cord neurons [3].

In addition, $\mathrm{T} \beta 4$ has wound-healing and tissue-repair properties, which is associated with its ability to inhibit apoptosis and inflammation [5-8]. It can promote dermal healing in normal animals as well as in impaired models of dermal wound healing, including aged mice, diabetic mice, and steroid-treated rats [5]. It promotes corneal wound healing by topical treatment after an alkali injury [6]. T $\beta 4$ inhibits polymorphonuclear leukocyte infiltration and suppresses the expression of interleukin proteins (IL-1 $\beta$ and IL-8), chemokines, macrophage inflammatory protein (MIP-1 $\alpha$, MIP-1 $\beta$, and MIP-2), and monocyte chemoattractant protein-1 (MCP-1) in corneal wound healing after an injury $[7,8]$. The anti-apoptotic properties displayed by $\mathrm{T} \beta 4$ is mediated by activating integrin-linked kinase (ILK), which results in activation of the survival kinase, Akt (also known as protein kinase B) $[2]$. 
Studies have shown that $\mathrm{T} \beta 4$ is up-regulated in regenerating muscle fibers after an injury [9]. Our previous study demonstrated that $\mathrm{T} \beta 4$ increased human skeletal myoblast (hSkM) migration, proliferation, and decreased cell injury under hypoxia [10]. The protective effect is associated with increased activities of PI3K and AKT, and reduced activities of caspase- 3 and -8 . The current study aims to investigate the therapeutic effects of $\mathrm{T} \beta 4$ for treatment of hind limb ischemia. This preliminary study suggests that localized delivery of $\mathrm{T} \beta 4$ increased angiogenesis, and increased skeletal muscle progenitor cell density in ischemic skeletal muscle, but failed to promote skeletal muscle regeneration.

\section{METHODS}

\subsection{Experimental Animal}

All animals were handled in compliance with the Guide for the Care and Use of Laboratory Animals approved by the Institutional Animal Care and Use Committee at the National University of Singapore (NUS), Singapore. All animals were maintained by research animal resource (RAR) of NUS.

Eight-week old female Balb/C mice were purchased from the Centre for Animal Resources of NUS and were housed in vented cage system with a 12 hour light-dark cycle and received standard mouse chow. Balb/c mouse is a laboratory-bred strain of the house mouse and is one of the most widely used inbred strains used in animal experimentation. Mouse hind limb ischemia model was produced in $19 \mathrm{Balb} / \mathrm{C}$ mice (18 - 20 grams body weight) by permanent ligation of femoral artery [11]. Briefly, the superficial and deep femoral arteries and internal iliac artery of the right hind limb were exposed and ligated from the inguinal ligament with the animal under anesthesia. Multiple ligatures of the artery beginning from the inguinal ligament down to the point where it bifurcates into the popliteal and saphenous arteries were closed with 8-0 nylon sutures. The superficial femoral artery was sectioned near and distal to the inguinal ligament between two suture ligations. The deep femoral artery was cauterized distal to its ligation site. Wounds were closed, and the animals were allowed to recover. All animals received antibiotics and painkiller post-surgery to prevent infection and relieve pain.

\section{2. $\mathrm{T} \beta 4$ Injection}

$\mathrm{T} \beta 4$ was dissolved in sterile saline to a concentration of $2 \mu \mathrm{g} / \mathrm{mL}$. Starting from the surgery day, $0.1 \mathrm{~mL}$ saline with $\mathrm{T} \beta 4$ was injected intramuscularly into the centre and periphery of femoral artery ligation area in the treatment group $(\mathrm{n}=10)$, while control animals received $0.1 \mathrm{~mL}$ saline injection $(\mathrm{n}=9)$. All animals received daily injection until 4 weeks after surgery. Then animals were sacrificed at 6 weeks after treatment and used for immunohistochemistry studies. No injection was made during the last two weeks. The aim of the additional two weeks was to exclude the immune reactions associated with injection, which may compromise the immunohistochemistry study of skeletal muscle.

\subsection{Histochemical and Immunohistochemical Studies}

Mouse medial thigh muscle was explanted. Cryo-sections of $6 \mu \mathrm{m}$ thickness were cut and used for fluorescence immunostaining. To quantify the muscle fibers with central nuclei, tissue sections were fluorescence immunostained with rabbit anti-dystrophin (Santa Cruz Biotech, USA) and 4,6-diamidino-2-phenylindole (DAPI, Sigma Aldrich, USA). To quantify the undifferentiated skeletal muscle progenitor cells, mouse tissue sections were dual immunostained with goat anti-Pax3/7 (Santa Cruz Biotech, USA) and rabbit anti-dystrophin. Eight non-over-laping microscopic fields/muscle $(\mathrm{n}=5$ animal each group) were calculated in control and $\mathrm{T} \beta 4$ group muscle sections.

\subsection{Vascular Density Quantification}

Dual fluorescence immunostaining for CD31 (Santa Cruz Biotech, USA) and smooth muscle actin (SMA) (Sigma Aldrich, USA) was carried out to quantify vascular density at 6 weeks after treatment. For each tissue section, the number of blood vessels was counted from 4 8 randomly selected microscopic fields at $200 \times$ magnification. A total of 25 tissue sections from five animals per group were used to measure the final average vascular density.

\subsection{Statistical Analysis}

All data are expressed as mean \pm SED. All statistical analyses were performed with SPSS (version 17). The difference between groups was analyzed by the method of student t-test. All tests were performed with a significance level of $5 \%$.

\section{RESULTS}

\subsection{Body Weight and Clinical Outcome}

There was no significant change of body weight between groups: they were $18.7 \pm 0.4 \mathrm{~g}$ and $19 \pm 0.4 \mathrm{~g}$ of control and $\mathrm{T} \beta 4$ group animals before treatment, respectively and increased to $19.6 \pm 0.5 \mathrm{~g}$ and $19.2 \pm 0.3 \mathrm{~g}$ after treatment. All mice survived the experiments. However, two mice in $\mathrm{T} \beta 4$ group each had one nail fall off from the ischemic limb. 


\subsection{Evidence for Neovascularization}

The vascular density based on CD31 immunostaining was significantly increased in $\mathrm{T} \beta 4$ group (562.5土 $\left.78.4 / \mathrm{mm}^{2}\right)$ as compared with control group (371.1 \pm $\left.125.7 / \mathrm{mm}^{2} ; p<0.05\right)$ at 6 weeks after treatment (Figures 1(A), (D) and (G)). The arteriole density based on CD31 and SMA dual immunostaining was similar between the T $\beta 4$ group $\left(\mathrm{T} \beta 4\right.$ group $\left.=27.2 \pm 16.9 / \mathrm{mm}^{2}\right)$ and control group $\left(35.3 \pm 6 / \mathrm{mm}^{2} ; p>0.05\right)$ (Figures 1(B), (C), (E), (F) and (H)). This suggests that $\mathrm{T} \beta 4$ increases angiogenesis only.

\subsection{Skeletal Muscle Histology}

Skeletal muscle histology demonstrated that $\mathrm{T} \beta 4$ insignificantly increased number of central nucleated fiber and central nuclei per fiber compared to control mouse (Figure 2). The numbers of central nuclei fiber were 8.9 \pm 2.1 and $9.5 \pm 1.6$, and the central nuclei per fiber were
$0.25 \pm 0.07$ and $0.48 \pm 0.09$ for control and $\mathrm{T} \beta 4$ groups, respectively. This suggests that $\mathrm{T} \beta 4$ did not increase skeletal muscle regeneration.

\subsection{Increased Pax $3 / 7^{+}$Skeletal Muscle Progenitor Cell Density}

Immunostaining for $\mathrm{Pax} 3 / 7$ expression showed that $\mathrm{T} \beta 4$ significantly increased $\mathrm{Pax} 3 / 7^{+}$nuclei $(13.7 \pm 2 \%)$ of total nuclei as compared to control group $(4.3 \pm 1.6 \%)$ per high magnification field $(p<0.05)$ (Figure 3). This suggests that $\mathrm{T} \beta 4$ increased the skeletal muscle progenitor cell density in ischemic skeletal muscle.

\section{DISCUSSION}

The current preliminary study demonstrates that $\mathrm{T} \beta 4$ increased angiogenesis and skeletal muscle progenitor cell density, but failed to promote skeletal muscle regeneration in ischemic skeletal muscle.
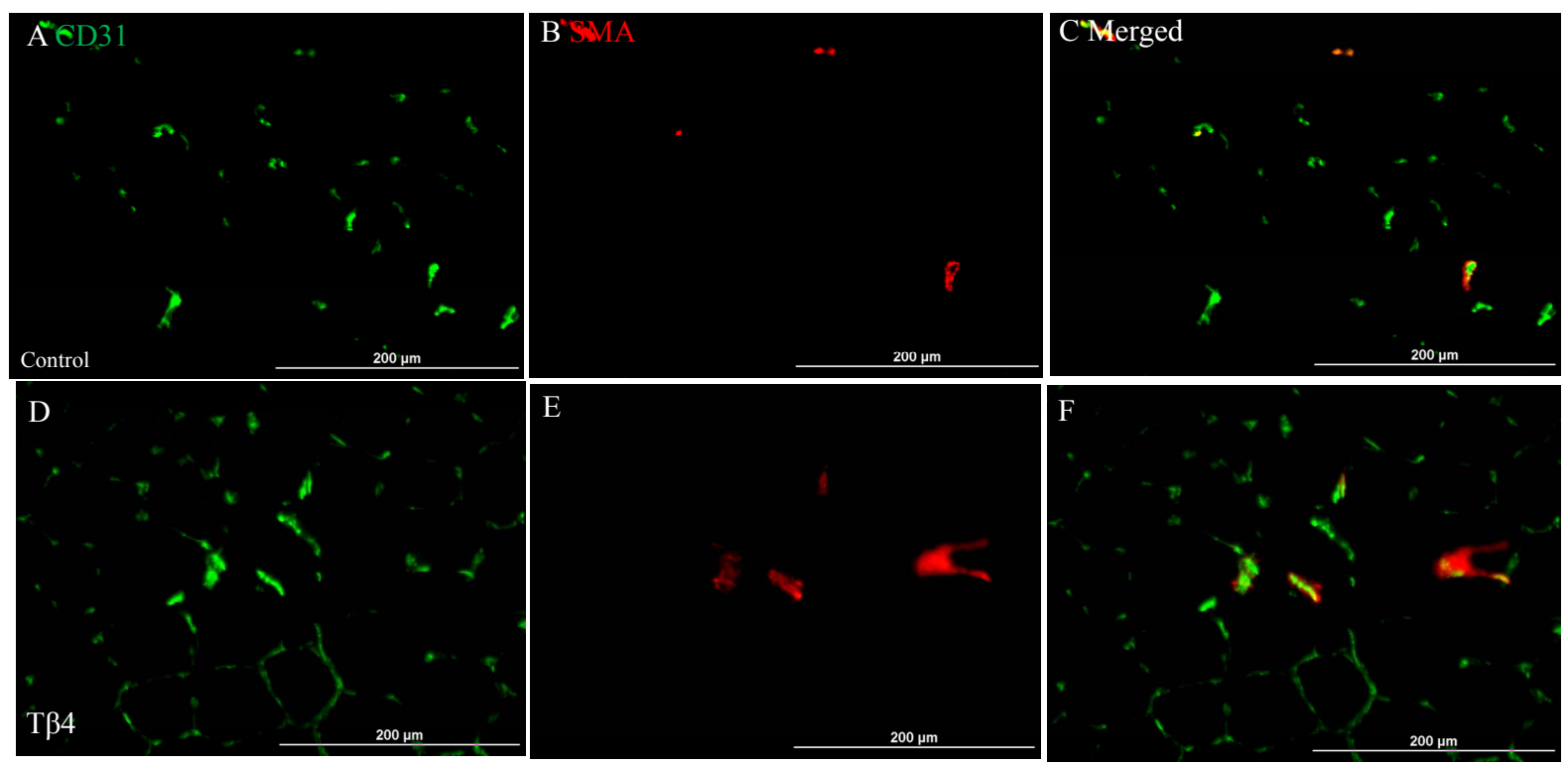

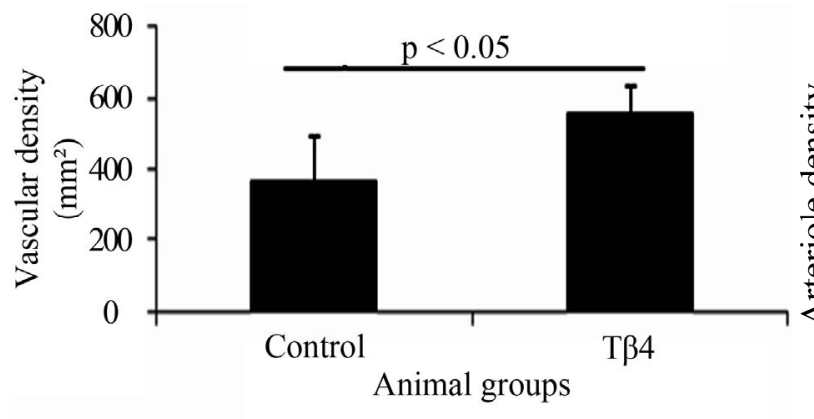

(G)

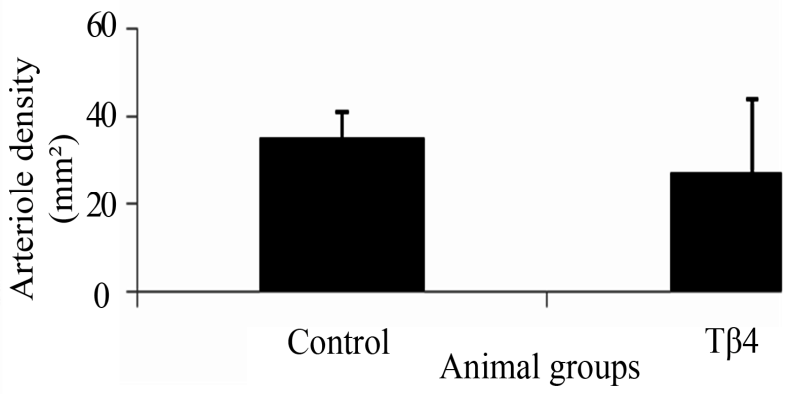

(H)

Figure 1. Dual fluorescent immunostaining for CD31 (A) and (D) and SMA (B) and (E) expressions. Merged images of CD31 and SMA from each group (C) and (F) were used to assess arteriole density. Quantification of total vascular (G) and arteriole densities (H). Significantly increased vascular density was found in T $\beta 4$ group as compared with control group based on CD31 expression. (Bar=200 $\mu \mathrm{m})$. 

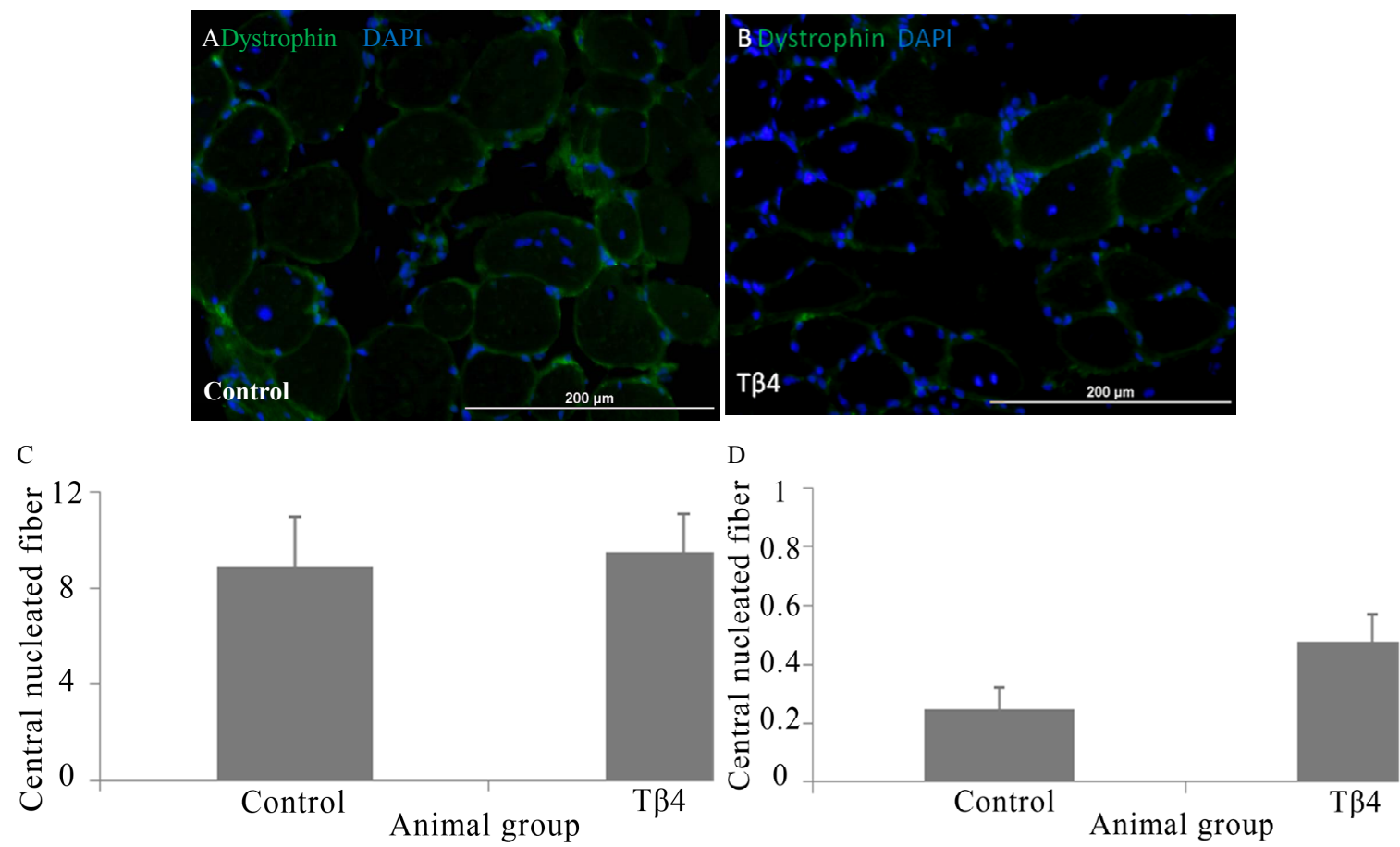

Figure 2. Skeletal muscle histology demonstrated that $\mathrm{T} \beta 4$ insignificantly increased numbers of central nucleated fiber and central nuclei per fiber. Pictures of mouse skeletal muscle showing central nucleated fibers of control (A) and T $\beta 4$ (B) groups. Quantification of central nucleated fiber (C) and central nuclei per fiber (D). (Bar $=200 \mu \mathrm{m})$.
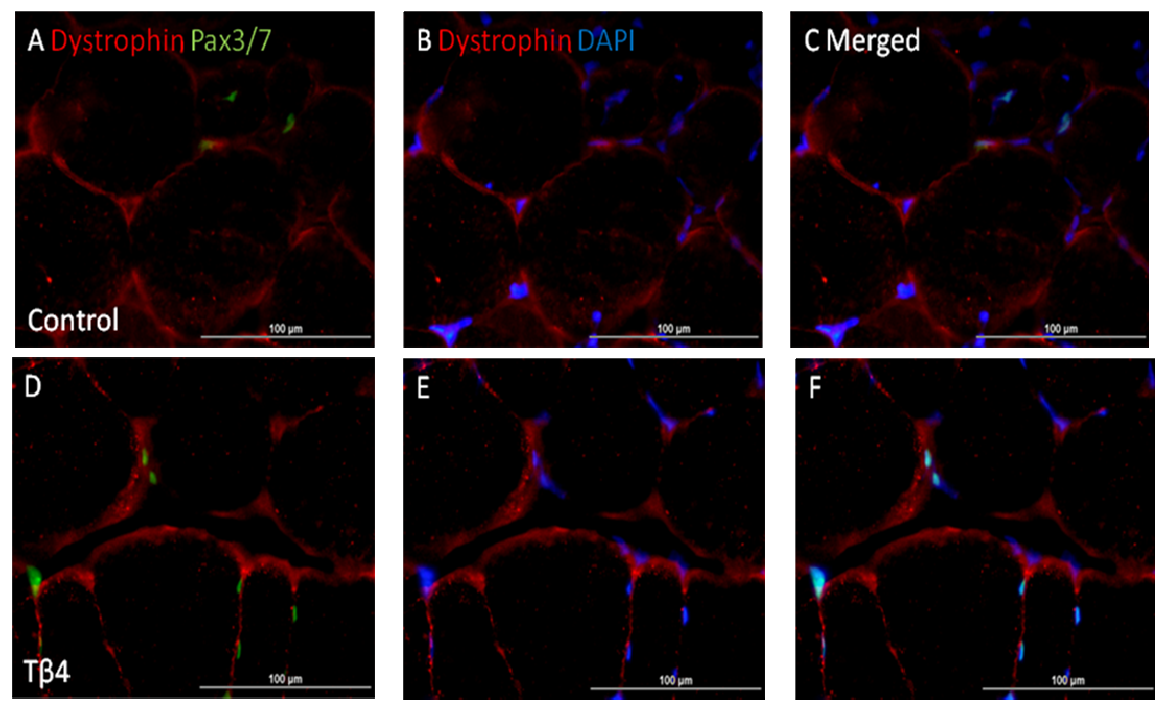

G

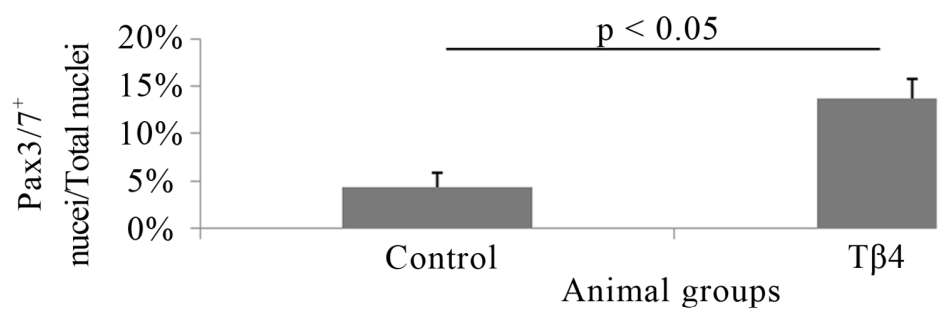

Figure 3. $\mathrm{T} \beta 4$ increased $\mathrm{Pax} 3 / 7^{+}$skeletal muscle progenitor cell density. Pictures of mouse skeletal muscle showing Pax $3 / 7^{+}$skeletal muscle progenitor cell of control (A)-(C) and T $\beta 4$ (D)-(F) groups. (G) Quantification of Pax3/7 $7^{+}$skeletal muscle progenitor cell density. $($ Bar $=100 \mu \mathrm{m})$. 
$\mathrm{T} \beta 4$ is the most abundant member of the $\beta$-thymosin family in mammalian tissue [1]. It is regarded as an intracellular monomeric G-actin sequestering peptide and inhibits the assembly of actin fibres within cells [1]. Besides, $\mathrm{T} \beta 4$ has been shown to play an important role in mediating cell migration, proliferation, and survival $[2,3,10]$. It promotes epicardial progenitor cells differentiation into endothelial cells for coronary vasculogenesis and angiogenesis [12]. T $\beta 4$ reduces cardiomyocyte injury after myocardial infarction and promotes cardiac cell migration and proliferation [2]. It enhances endothelial cell migration and adhesion [13-15]. Thus, $\mathrm{T} \beta 4$ has been shown to promote tissue regeneration [14-16].

Our previous study demonstrated that $\mathrm{T} \beta 4$ increased human skeletal myoblast (hSkM) migration, proliferation, and decreased cell injury under hypoxia in vitro [10]. The current study further tested the beneficial effects of $\mathrm{T} \beta 4$ on neovascularization and muscle regeneration in a mouse model of ischemia limb disease. It was found that $\mathrm{T} \beta 4$ significantly increased capillary density. This is associated with its biological function on endothelial cells: promoting endothelial cell migration and proliferation. Malinda et al., [13] demonstrated that $\mathrm{T} \beta 4$ has chemoattractive activity and promotes angiogenesis by stimulating the migration of endothelial cells in vitro and in vivo as well. Grant et al. [15] showed that T $\beta 4$ not only increased tubular structure formation of HUVECs in vitro, but also enhanced vascular sprouting in the coronary artery ring angiogenesis assay. Qiu et al. [16] found that $\mathrm{T} \beta 4$ induced endothelial cell migration through the $\mathrm{PI} 3 \mathrm{~K} / \mathrm{Akt} / \mathrm{eNOS}$ signal pathway. Current study is consistent with these studies. It was found that $\mathrm{T} \beta 4$ administration increased capillary density by $50 \%$ as compared with control animal based on CD31 expression. However, no significant increase of arteriole density was noted. Thus, $\mathrm{T} \beta 4$ stimulates angiogenesis but not arteriogenesis in mouse ischemic limb.

Significantly increased Pax $3 / 7$ expressing nuclei were found in $\mathrm{T} \beta 4$ treated skeletal muscle. This indicates that $\mathrm{T} \beta 4$ increased Pax $3 / 7$ expressing progenitor cell density in ischemic limb muscle. Recent studies have identified $\mathrm{Pax}^{+} / \mathrm{Pax} 7^{+}$somatic cells as the source of muscle progenitors $[17,18]$. $\mathrm{Pax}^{+} / \mathrm{Pax}^{+}$cells are the common progenitors responsible for all embryonic, fetal, and adult myogenesis in axial and limb muscles. Pax3 is sufficient to induce MyoD and Myf5 in vitro [19]. Pax7 is required for maintenance of adult satellite cells $[20,21]$. In the current study, a significantly increased level of Pax $3 / 7^{+}$ cells was found. T $\beta 4$ may stimulate the proliferation of $\mathrm{Pax} 3 / 7^{+}$progenitor cells in ischemic area or enhance the migration of cells to ischemic muscle. Our previous study demonstrated that $\mathrm{T} \beta 4$ increased hSkM migration and proliferation in vitro. Thus, it's possible that $\mathrm{T} \beta 4$ increases $\mathrm{Pax} 3 / 7^{+}$progenitor cell proliferation and sti- mulates cell migration and homing to ischemic muscle.

Though Pax3/7 progenitor cell density was increased, there is no significantly increased skeletal muscle regeneration between $\mathrm{T} \beta 4$ and control groups. The central nucleated fiber and the central nuclei per fiber of $\mathrm{T} \beta 4$ were insignificantly increased as compared with control group. This finding is inconsistent with Spurney's study [22], which demonstrated that $\mathrm{Mdx}$ mice treated with $\mathrm{T} \beta 4$ showed a significant increase in skeletal muscle regenerating fibers compared to untreated Mdx mice. This might be related to animal model and dosage of T $\beta 4$. Spurney's study used a chronic muscle degeneration animal model and injected $\mathrm{T} \beta 4$ twice per week for six months. The current study adopted an acute ischemic model and daily injected T $\beta 4$ for four weeks. The different pathophysiological condition of skeletal muscle may have a different response to $\mathrm{T} \beta 4$. A much higher dose of $\mathrm{T} \beta 4$ was used in Spurney's study also. This may stimulate the fusion of skeletal muscle progenitor cells, which in turn contributes to the muscle regeneration.

In summary, the current study demonstrates that $\mathrm{T} \beta 4$ stimulates angiogenesis and increased skeletal muscle progenitor cell density, but failed to increase regenerating muscle fiber in ischemic limb muscle. Further study should be performed to investigate the underlying mechanism that the role of $\mathrm{T} \beta 4$ on homing and migrating of Pax $3 / 7^{+}$muscle progenitor cells has.

\section{ACKNOWLEDGEMENTS}

The project was funded by Singapore National Medical Research Council (NMRC) grant EDG09may058.

\section{REFERENCES}

[1] Pollard, T.D. and Borisy, G.G. (2003) Cellular motility driven by assembly and disassembly of actin filaments. Cell, 112, 453-465. doi:10.1016/S0092-8674(03)00120-X

[2] Bock-Marquette, I., et al. (2004) Thymosin $\beta 4$ activates integrin-linked kinase and promotes cardiac cell migration, survival and cardiac repair. Nature, 432, 466-472. doi:10.1038/nature03000

[3] Yang, H., et al. (2008) The promotive effects of thymosin $\beta 4$ on neuronal survival and neurite outgrowth by upregulating L1 expression. Neurochemical Research, 33, 2269-2280. doi:10.1007/s11064-008-9712-y

[4] Philp, D., et al. (2004) Thymosin $\beta 4$ increases hair growth by activation of hair follicle stem cells. The FASEB Journal, 18, 385-387.

[5] Philp, D., Scheremeta, B., Sibliss, K., Zhou, M., Fine, E.L., Nguyen, M., et al. (2006) Thymosin $\beta 4$ promotes matrix metalloproteinase expression during wound repair. Journal of Cellular Physiology, 208, 195-200. doi: $10.1002 /$ jep. 20650

[6] Sosne, G., et al. (2005) Thymosin $\beta 4$ modulates corneal matrix metalloproteinase levels and polymorphonuclear 
cell infiltration after alkali injury. Investigative Ophthalmology \& Visual Science, 46, 2388-2395. doi:10.1167/iovs.04-1368

[7] Qiu, P., et al. (2011) Thymosin $\beta 4$ inhibits TNF-alphainduced NF- $\kappa \mathrm{B}$ activation, IL- 8 expression, and the sensitizing effects by its partners PINCH-1 and ILK. The FASEB Journal, 25, 1815-1826. doi:10.1096/fj.10-167940

[8] Sosne, G., et al. (2005) Thymosin $\beta 4$ modulates corneal matrix metalloproteinase levels and polymorphonuclear cell infiltration after alkali injury. Investigative Ophthalmology \& Visual Science, 46, 2388-2395. doi:10.1167/iovs.04-1368

[9] Ferre, P.J., et al. (2007) Longitudinal analysis of gene expression in porcine skeletal muscle after post-injection local injury. Pharmaceutical Research, 24, 1480-1489. doi:10.1007/s11095-007-9266-8

[10] Ye, L., Su, L.P., Pi, W.F. and Law, P.K. (2012) Role of thymosin $\beta 4$ on skeletal myoblast migration, proliferation, and survival. Recent Patents on Regenerative Medicine, 2, 146-155. doi: $10.2174 / 2210297311202020146$

[11] Ye, L., et al. (2010) Liposome-based vascular endothelial growth factor-165 transfection with skeletal myoblast for treatment of ischaemic limb disease. Journal of Cellular and Molecular Medicine, 14, 323-336. doi:10.1111/j.1582-4934.2008.00454.x

[12] Smart, N., et al. (2007) Thymosin $\beta 4$ is essential for coronary vessel development and promotes neovascularization via adult epicardium. Annals of the New York Academy of Science, 1112, 171-188. doi:10.1196/annals. 1415.000

[13] Malinda, K.M., Goldstein, A.L. and Kleinman, H.K. (1997) Thymosin $\beta 4$ stimulates directional migration of human umbilical vein endothelial cells. The FASEB Journal, 11, 474-481.
[14] Chiu, L.L. and Radisic, M. (2011) Controlled release of thymosin $\beta 4$ using collagen-chitosan composite hydrogels promotes epicardial cell migration and angiogenesis. Journal of Controlled Release, 155, 376-385. doi:10.1016/j.jconrel.2011.05.026

[15] Grant, D.S., et al. (1999) Thymosin $\beta 4$ enhances endothelial cell differentiation and angiogenesis. Angiogenesis, 3, 125-135. doi:10.1023/A:1009041911493

[16] Qiu, F.Y., et al. (2009) Thymosin $\beta 4$ induces endothelial progenitor cell migration via PI3K/Akt/eNOS signal transduction pathway. Journal of Cardiovascular Pharmacology, 53, 209-214. doi:10.1097/FJC.0b013e318199f326

[17] Kassar-Duchossoy, L., et al. (2005) Pax3/Pax7 mark a novel population of primitive myogenic cells during development. Genes \& Development, 19, 1426-1431. doi:10.1101/gad.345505

[18] Relaix, F., et al. (2005) A Pax3/Pax7-dependent population of skeletal muscle progenitor cells. Nature, 435, 948953. doi:10.1038/nature03594

[19] Maroto, M., et al. (1997) Ectopic Pax-3 activates MyoD and Myf-5 expression in embryonic mesoderm and neural tissue. Cell, 89, 139-148. doi:10.1016/S0092-8674(00)80190-7

[20] Kuang, S., et al. (2006) Distinct roles for Pax7 and Pax3 in adult regenerative myogenesis. Journal of Cell Biology, 172, 103-113. doi:10.1083/jcb.200508001

[21] Relaix, F., et al. (2006) Pax3 and Pax7 have distinct and overlapping functions in adult muscle progenitor cells. Journal of Cell Biology, 172, 91-102. doi: $10.1083 /$ jcb. 200508044

[22] Spurney, C.F., et al. (2010) Evaluation of skeletal and cardiac muscle function after chronic administration of thymosin $\beta 4$ in the dystrophin deficient mouse. PLOS One, 5, p. e8976. doi:10.1371/journal.pone.0008976 